\title{
A Simple Method for Calculating the Deflection of the Vertical From Gravity Anomalies, with its Applications to 16 Selected Stations in U. S. A.
}

\author{
By \\ Chuji Tsubor* and Akio Hayatu \\ Department of Geophysics Faculty of Science, Tolyo University \\ Tokyo, Japan
}

$\S 1$. To calculate the $\xi$ - and $\eta$-components of the deflection of the vertical at a point situated on the earth's surface from gravity anomalies which are known on the same surface is one of the classical problems in physical geodesy since the day of C.G. STokms. The well-known formula which was derived by STokes for this purpose is theoretically beautiful but unfortunately it cannot be used in its original form at present because of our rather poor knowledge concerning the gravity anomaly distribution all over the world, which is essential for the application of that method. Among the many efforts which have since been made to make this method of Srokes competent for practical applications, the derivation of a useful formula by Vening Mrornesz (1928) is to be particularly mentioned. In this formula, the effect of the gravity anomaly in an elementary compartment of the earth's surface upon the deflection of the vertical at a certain point on the same surface is given in terms of the angular distance $\psi$ of the compartment from the point and of its azimuth $\theta$ as seen from the same point. The total deflection is obtained if such an elementary effect is integrated over the entire surface of the earth, that is, from 0 to $\pi$ with respect to $\psi$ and from 0 to $2 \pi$ with respect to $\theta$.

Solnin's table (1947) which was published later is an important contribution to this field of study. In this table are given numerical values of the quantities needed if one wishes to apply Mernesz's formula to actual problems.
Making use of this table, Donald A. RICE of U. S. Coast and Geodetic Survey (1952) calculated the $\xi$-and $\eta$-components of the deflections of the vertical at 16 stations selected in U.S.A. using free-air anomalies known around them. Rrce gave curves showing how the calculated $\xi$ and $\eta$ tend to their respective definite values according as the limit of integration with respect to $\psi$ is successively increased. Looking at these interesting curves of RicE, it was noticed that the greatest distance talen by RICE in the direction of $\psi$ was a little over $600 \mathrm{~km}$ at most, which is only a small fraction of the earth's radius. So far as RICE's calculations are concerned, therefore, no appreciable part seems to have been played by the assumption of the sphericity of the earth which underlies Mernesz's formula. It naturally occurred to the present writers that it will be interesting to see what will happen if the sphericity of the earth is ignored altogether in this problem. There can be two ways of approach to investigate this, say (A) and (B): the one (A) to solve the plane problen from the outset, while the other (B) to find the approximate expression of Meinesz's formula in case when $\psi$ is made small. It might well be expected that the two will turn to the same formula after all and that this formula when applied to the actual problems in U.S. A. will give results that differ little from those obtained by Rice with much higher approximations.

§2. First, we shall follow the way (A). Taking the cylindrical co-ordinate system with its origin at the point at which the

* The preliminary report was published in Proc. Japan Acad., 30 (1954), 461. 
deflection is to be calculated, the gravity potential $U$ (anomalous part only) can be expressed by a Fourier-Bessel series such as

$$
\begin{aligned}
U & =\sum_{n} \int_{0}^{\infty} \frac{B_{n k}^{c}}{k} J_{n}(k r) \exp (-k z) d k \cdot \cos n \theta \\
& +\sum_{n} \int_{0}^{\infty} \frac{B_{n k}^{s}}{k} J_{n}(k r) \exp (-k z) d k \cdot \sin n \theta \ldots
\end{aligned}
$$

using customary notations. From (1), the gravity anomaly $\Delta g$ at any $r, \theta$, and $z$ is given by

$$
\begin{aligned}
\Delta g & =-\frac{\partial U}{\partial z}=\sum_{n} \int_{0}^{\infty} B_{n k}^{c} J_{n}(k r) \exp (-k z) d k \cdot \cos n \theta \\
& +\sum_{n} \int_{0}^{\infty} B_{n k}^{s} J_{n}(k r) \exp (-k z) d k \cdot \sin n \theta . \ldots(2)
\end{aligned}
$$

Since $z=0$ at the earth's surface, (2) reduces into

$$
\begin{aligned}
\Delta g=\sum_{n} \int_{0}^{\infty} B_{n k}^{c} J_{n}(k r) d k \cdot \cos n \theta \\
\quad+\sum_{n} \int_{0}^{\infty} B_{n k}^{s} J_{n}(k r) d k \cdot \sin n \theta \\
=\int_{0}^{\infty} B_{0 k}^{c} J_{0}(k r) d k \\
\quad+\int_{0}^{\infty} B_{1 k}^{c} J_{1}(k r) d k \cdot \cos \theta \\
+\int_{0}^{\infty} B_{2 k}^{c} J_{2}(k r) d k \cdot \cos 2 \theta+\cdots \\
\quad+\int_{0}^{\infty} B_{1 k}^{s} J_{1}(k r) d k \cdot \sin \theta \\
\quad+\int_{0}^{\infty} B_{2 k}^{s} J_{2}(k r) d k \cdot \sin 2 \theta+\cdots \\
\equiv C_{0}(r)+C_{1}(r) \cos \theta+C_{2}(r) \cos 2 \theta+\cdots \\
+S_{1}(r) \sin \theta+S_{2}(r) \sin 2 \theta+\cdots
\end{aligned}
$$

where $C_{0}(r) \equiv \int_{0}^{\infty} B_{0 k}^{s} J_{0}(k r) d k$,

$$
\begin{aligned}
& C_{1}(r) \equiv \int_{0}^{\infty} B_{1 k}^{c} J_{1}(k r) d k, \\
& S_{1}(r) \equiv \int_{1}^{\infty} B_{1 k}^{s} J_{1}(k r) d k,
\end{aligned}
$$

On the other hand, the $\xi$-and $\eta$-components of the deflection of the vertical at the origin are given if $r$ in the following expressions, (4) and (5), is made to tend to 0.

$$
\xi=\frac{1}{g}\left(\frac{\partial U}{\partial r}\right)_{\substack{\begin{subarray}{c}{c=0 \\
z=0} }} \\
{0}\end{subarray}}
$$

$$
=\frac{1}{g}\left\{\int_{0}^{\infty} B_{0 k}^{\mathrm{c}} J_{0}^{\prime}(k r) d k+\int_{0}^{\infty} B_{1 k}^{\mathrm{c}} J_{1}{ }^{\prime}(k r) d k+\cdots\right\},
$$

$$
\begin{aligned}
\eta & =\frac{1}{g}\left(\frac{\partial V}{\partial r}\right)_{\substack{\theta=\pi / 2 \\
z=0}} \\
& =\frac{1}{g}\left\{\int_{0}^{\infty} B_{1 k}^{s} J_{1}^{\prime}(k r) d k+\cdots \cdots\right\} .
\end{aligned}
$$

If, in doing this, the following mathematical relations concerning BESSEL functions are noticed,

$$
J_{1}^{\prime}(0)=\frac{1}{2}, \quad J_{1}^{\prime}(0)=J_{2}^{\prime}(0)=\cdots=0,
$$

the following expressions are obtained,

$$
\begin{aligned}
& \xi=\frac{1}{2 g} \int_{0}^{\infty} B_{1 k}^{c} d k, \\
& \eta=\frac{1}{2 g} \int_{0}^{\infty} B_{1 k}^{s} d k,
\end{aligned}
$$

$B_{1 k}^{c}$ and $B_{1 k}^{s}$ being the constants which already appeared in the expression (3). The next task then is to evaluate the integrals in (7) and (8). If the following mathematical relation again concerning a Besser function is noticed,

$$
\int_{0}^{\infty} \frac{J_{1}(k r)}{r} d r=1
$$

the integration with respect to $k$ can be replaced by that with respect to $r$, thus

$$
\begin{gathered}
\int_{0}^{\infty} B_{1 k}^{c} d k=\int_{0}^{\infty} \int_{0}^{\infty} B_{1 k}^{\mathrm{c}} \frac{J_{1}(k r)}{r} d k d r \\
=\int_{0}^{\infty} \frac{C_{1}(r)}{r} d r,
\end{gathered}
$$

and similarly

$$
\int_{0}^{\infty} B_{1 k}^{s} d k=\int_{0}^{\infty} \frac{S_{1}(r)}{r} d r
$$

The final expressions for $\xi$ and $\eta$ are therefore simply

$$
\begin{aligned}
& \xi=\frac{1}{2 g} \int_{0}^{\infty} \frac{C_{1}(r)}{r} d r, \\
& \eta=\frac{1}{2 g} \int_{0}^{\infty} \frac{S_{1}(r)}{r} d r .
\end{aligned}
$$

These expressions state that what are needed for our purpose are only $C_{1}(r)$ and $S_{1}(r)$ which are the first order coefficients when the 
gravity anomalies along a circle $r=r$ drawn around the origin are expressed by the Fourier series of azimuth. So our method for calculating $\xi$ and $\eta$ practically consists in :

1) drawing a series of concentric circles around the point for which $\xi$ and $\eta$ are to be calculated,

2) reading the values of $\Delta g$ along each of the circles at a reasonably many number of points,

3) calculating the first order coefficients $C_{1}(r)$ and $S_{\perp}(r)$ for expressing $\Delta g$ along each of the circles by the Fourrer series of azimuth,

4) dividing $C_{1}(r)$ and $S_{1}(r)$ by corresponding $r$,

5) integrating $C_{1}(r) / r$ and $S_{1}(r) / r$ with respect to $r$ from 0 to $\infty$ and lastly,

6) dividing the integrals by $2 g$.

The integrals $\int_{0}^{\infty} \frac{C_{1}(r)}{r} d r$ and $\int_{0}^{\infty} \frac{S_{1}(r)}{r} d r$ may first appear to diverge at $r=0$, since $r$ is contained in the denominators of the integrands. But this is not actually the case, because $C_{1}(r)$ and $S_{1}(r)$ themselves tend to 0 also as the first power of $r$ if $r$ tends to 0 . If $r$ is small, $C_{1}(r)$ is a linear function of $r$ and may be written as

$$
C_{1}(r)=\alpha r, \quad 0 \leqq r \leqq R,
$$

where $R$ is the limit up to which $C_{1}(r)$ may be regarded to change linearly as $a r$. The integral from 0 to $\infty$ may be split into two pars : the one from 0 to $R$ and the other from $R$ to $\infty$, and may be written as

$$
\begin{aligned}
\xi & =\frac{1}{2 g} \int_{0}^{\infty} \frac{C_{1}(r)}{r} d r \\
& =\frac{1}{2 g}\left\{\int_{0}^{R} \frac{C_{1}(r)}{r} d r+\int_{n}^{\infty} \frac{C_{1}(r)}{r} d r\right\} \\
& =\frac{1}{2 g}\left\{\alpha R+\int_{n}^{\infty} \frac{C_{1}(r)}{r} d r\right\} \\
& =\frac{1}{2 g}\left\{C_{1}(R)+\int_{R}^{\infty} \frac{C_{1}(r)}{r} d r\right\} .
\end{aligned}
$$

The value of $\xi$ as given by (15) is evidently finite. The splitting of the integrals into the two parts, however, need not be necessarily exactly at the very limit up to which $C_{1}(r)$ changes linearly with $r$. If, by any method, $\xi\left(R^{\prime}\right)$ is known to be the total contribution of $\Delta g$ within $r=R^{\prime}, \xi$ may be written as

$$
\xi=\xi\left(R^{\prime}\right)+\frac{1}{2 g} \int_{R^{\prime}}^{\infty} \frac{C_{1}(r)}{r} d r,
$$

where $R^{\prime}$ may take any value. Also

$$
\eta=\eta\left(R^{\prime}\right)+\frac{1}{2 g} \int_{R^{\prime}}^{\infty} \frac{S_{1}(r)}{r} d r . \cdot
$$

§3. Now in order to investigate its feasibility, our new method will be applied to calculate the values of $\xi$ and $\eta$ at the same 16 points in U.S. A. for which Rrce's values have been known. The comparison of the values by RICE and by the present writers will make it clear how far the new method can safely be used.

On the map of $\Delta g_{0}$ published by Rrce, concentric circles were drawn at every $20 \mathrm{~km}$ distance interval around the point at which $\xi$ and $\eta$ are to be calculated. $C_{1}(r)$ and $S_{1}(r)$ were calculated from 24 equidistant readings of $\Delta g_{0}$ along each of the circles around the origin by the ordinary harmonic analysis method. As to the values of $\xi(20)$ and $\eta(20)$, however, no accurate estimations have been possible for us for the map published by RICE was unfortunately of too large a scale to admit them. What could be done was only to calculate $\frac{1}{2 g} \int_{20}^{\infty} \frac{C_{1}(r)}{r} d r$ and $\frac{1}{2 g} \int_{20}^{\infty} \frac{S_{1}(r)}{r} d r$, according to (16) and (17), and to the calculated values to add the values of $\xi(20.09)$ and $\eta(20.09)$ ob- $^{-}$ tained by RICE. At the writers' request, the values of $\xi(20.09)$ and $\eta(20.09)$ were kindly supplied by Rice. He sent also the values for larger distances for reference.

In order to demonstrate the procedures followed in our calculations, the results for Meades Ranch, one of the 16 stations, are given in Table I as an example.

Since the integrands in (16) and (17) contain $r$ in the demominators and $C_{1}(r)$ and $S_{1}(r)$ do not increase systematically at large $r$, the integrals decrease with $r$. Owing to this reason, the values of $\int_{20}^{r} \frac{C_{1}(r)}{r} d r$ and $\int_{20}^{r} \frac{S_{1}(r)}{r} d r$ do not change much at larger $r$, so that the 
integrals from 0 to $\infty$ can be approximated by those from 0 to a reasonably large $r$. If $\xi(r)$ and $\eta(r)$ have the following meaning :

$$
\begin{aligned}
& \xi(r)=\xi(20)+\frac{1}{2 g} \int_{20}^{r} \frac{C_{1}(r)}{r} d r, \\
& \eta(r)=\eta(20)+\frac{1}{2 g} \int_{20}^{r} \frac{S_{1}(r)}{r} d r,
\end{aligned}
$$

Table II will show the values of $\xi(r)$ and $\eta(r)$ found by our calculations for all the 16 points. The values by RICE are also entered in the table for the sake of comparison and are printed in gothic type.

TABLE I

Meades Ranch

$\xi$-Component

\begin{tabular}{r|r|r|r|r|c}
\hline$r(\mathrm{~km})$ & $\begin{array}{c}C_{1}(r) \\
(\mathrm{mag})\end{array}$ & $\begin{array}{c}C_{1}(r) / r \\
10^{-9}\end{array}$ & $\begin{array}{c}\int_{20}^{r} \frac{C_{1}(r)}{r} d r \\
10^{-3}\end{array}$ & $\begin{array}{c}\xi \\
\text { (second) }\end{array}$ & $\begin{array}{c}\xi \\
\text { (second) }\end{array}$ \\
\hline 0 & & & & & \\
20 & 0.33 & 0.17 & - & -1 & $-0.28^{*}$ \\
40 & 6.42 & 1.61 & 1.77 & 0.19 & -0.09 \\
60 & 6.40 & 1.07 & 4.44 & 0.47 & 0.19 \\
80 & 2.26 & 0.28 & 5.79 & 0.61 & 0.33 \\
100 & 3.81 & 0.38 & 6.46 & 0.68 & 0.40 \\
120 & 1.12 & 0.09 & 6.93 & 0.73 & 0.45 \\
140 & -2.86 & -0.20 & 6.82 & 0.72 & 0.44 \\
160 & -2.76 & -0.17 & 6.44 & 0.68 & 0.40 \\
180 & -4.08 & -0.23 & 6.05 & 0.64 & 0.36 \\
200 & -7.34 & -0.37 & 5.45 & 0.57 & 0.29 \\
220 & -10.78 & -0.49 & 4.60 & 0.48 & 0.20 \\
\hline
\end{tabular}

* RICE's value

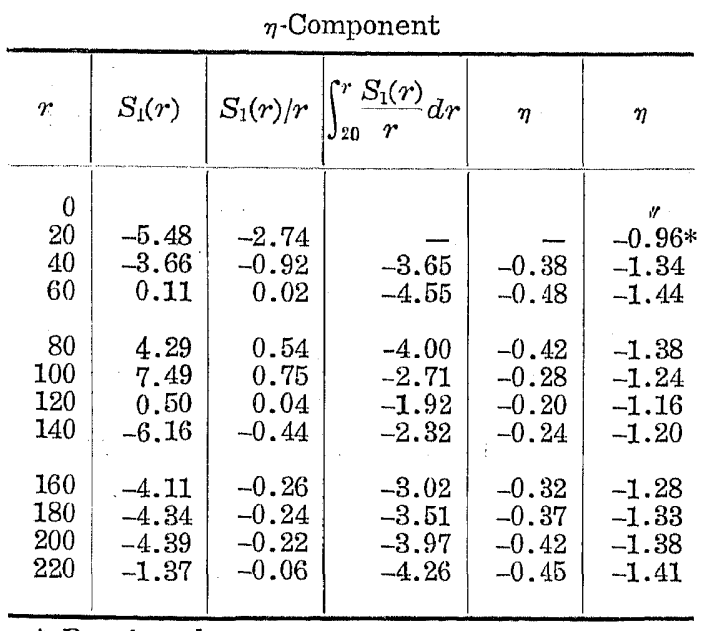

* RICE's value

The values of $\xi(r)$ and $\eta(r)$ are plotted in
Fig. 1 against $r$ up to which integrations were extended. It is clearly seen that the values of RICE and ours agree satisfactorily well. At a few points, Little Rock Longitude for instance, however, there is some systematic difference between the two which amounts to $0.3^{\prime \prime}$. The origin of such a difference is not yet clear.

$\S 4$. Now the second way of approach (B) will be taken to see what will happen if $\psi$ is made small in Meinesz's formula. The formulas as given by Mernesz are as follows:

$$
\xi=-\frac{1}{2 \pi g} \int_{0}^{2 \pi} \int_{0}^{\pi} \Delta g(\psi \theta) \frac{d f(\psi)}{d \psi} \sin \psi \cos \theta d \psi d \theta,
$$$$
\eta=-\frac{1}{2 \pi g} \int_{0}^{2 \pi} \int_{0}^{\pi} \Delta g(\psi \theta) \frac{d f(\psi)}{d \psi} \sin \psi \sin \theta d \psi d \theta,
$$
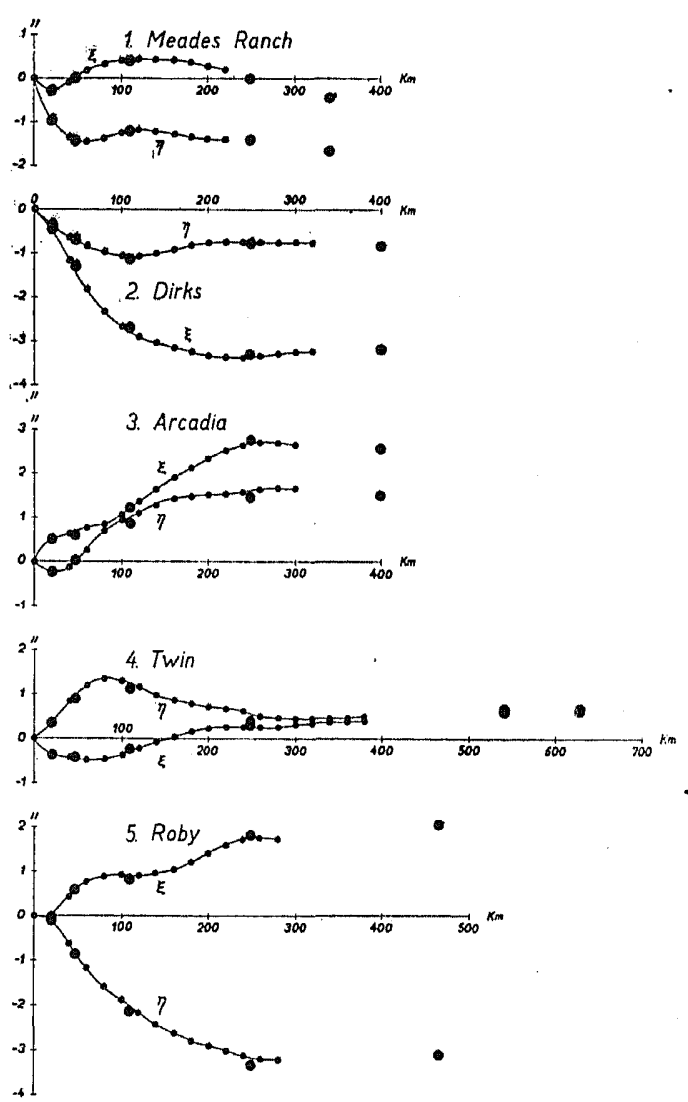

Fig. 1 
A Simple Method for Calculating the Deflection of the Vertical, etc.
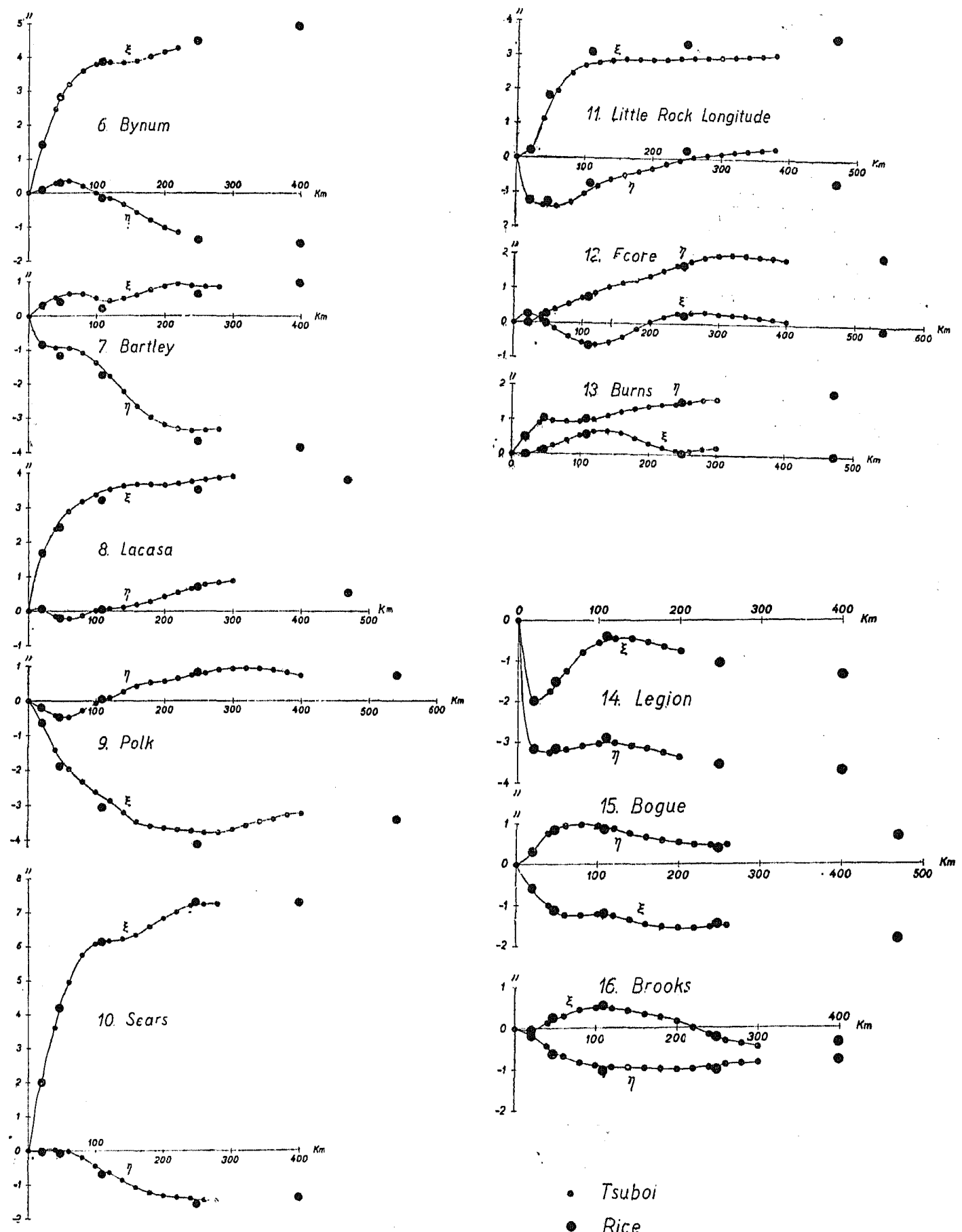

- Tsuboi

- Rice

Fig. 1. 


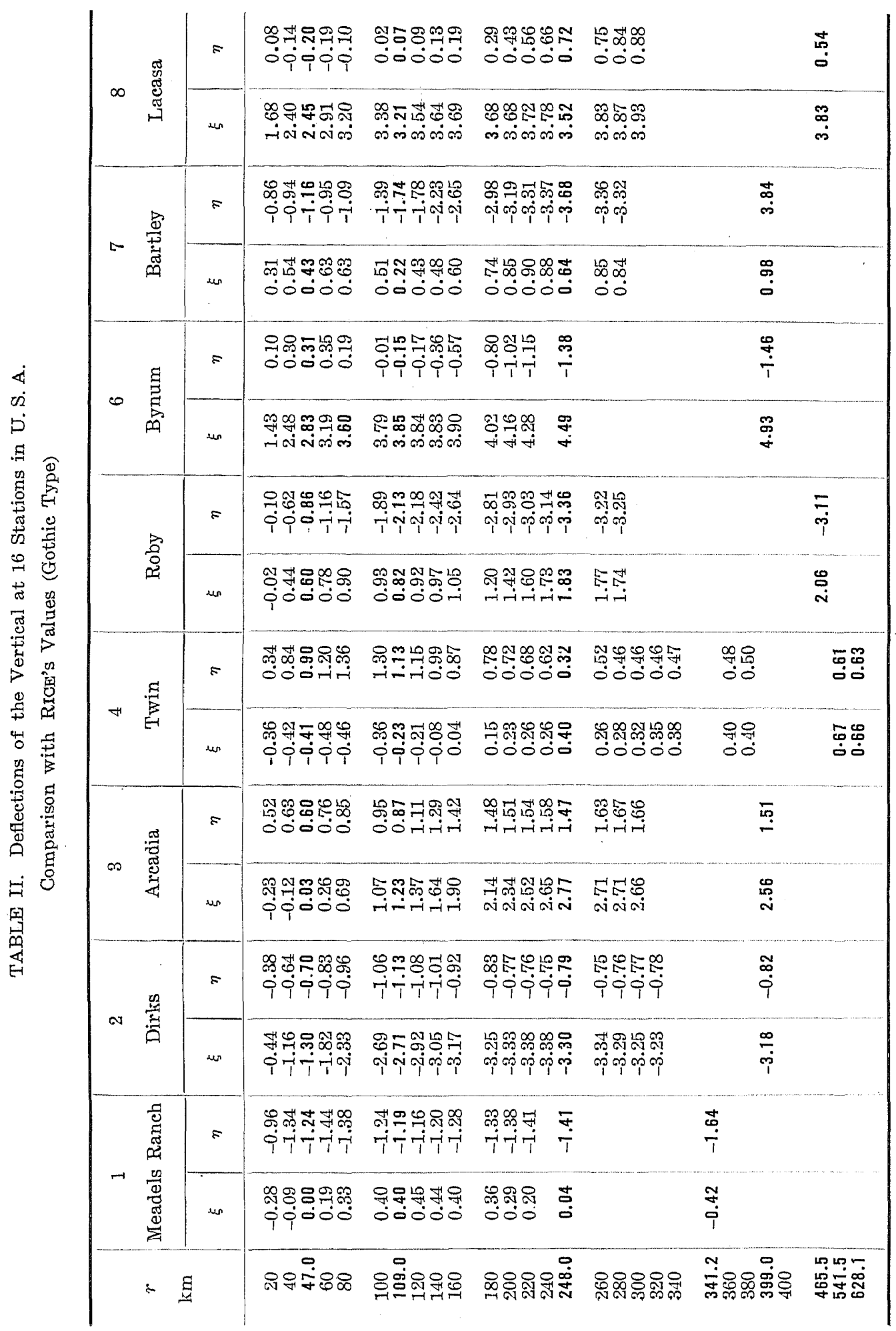


A Simple Method for Calculating the Deflection of tne Vertical, etc.

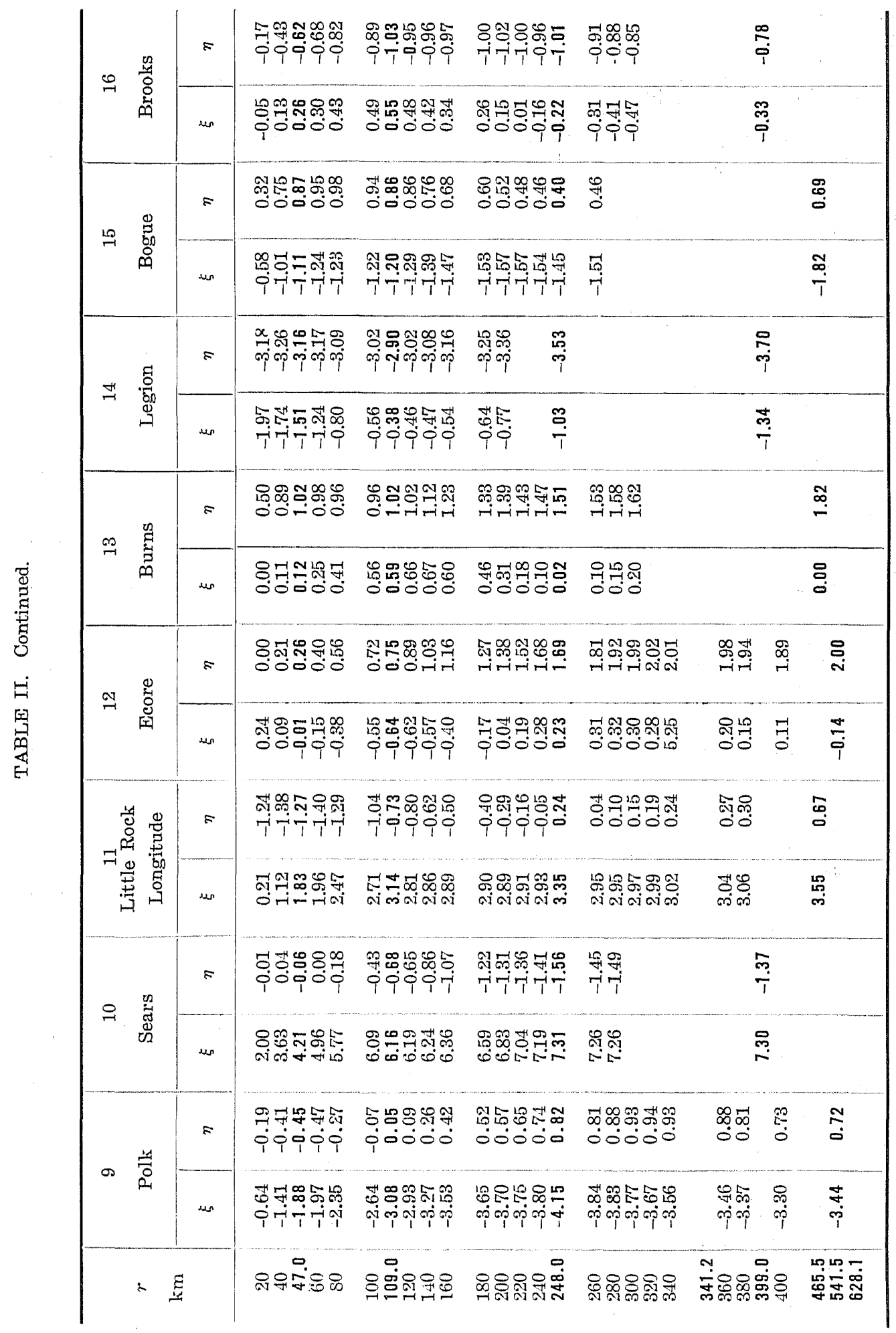




$$
\begin{aligned}
\frac{d f}{d \psi}= & \frac{1}{2}\left[-\frac{\cos \psi / 2}{2 \sin 2 \psi / 2}-3 \cos \frac{2}{\psi}+5 \sin \psi\right. \\
& +3 \sin \psi \log \left(\sin \frac{\psi}{2}+\sin ^{2} \frac{\phi}{2}\right) \\
& \left.-\frac{3}{2} \frac{1+2 \sin \psi / 2}{1+\sin \psi} \cot \frac{\psi}{2} \cos \psi\right] \ldots
\end{aligned}
$$

in which $\psi$ is the angular distance between a compartment on the earth's sruface where $\Delta g$ is known and the point at which the deflection of the vertical is to be calculated. $\theta$ is the azimuth of the direction that connect the two.

As already stated by Sollins (1947), if $\psi$ is small, (22) can be written approximately as

$$
\frac{d f}{d \psi}=-\frac{1}{\psi^{2}}-\frac{3}{2 \psi} .
$$

Therefore

$$
\xi=\frac{1}{2 \pi g} \int_{0}^{2 \pi} \int_{0}^{\pi} \Delta g(\psi \theta) \frac{1}{\psi} \cos \theta d \psi d \theta,
$$

and

$$
\eta=\frac{1}{2 \pi g} \int_{0}^{2 \pi} \int_{0}^{\pi} \Delta g(\psi \theta) \frac{1}{\psi} \sin \theta d \psi d \theta .
$$

But since

$$
\frac{1}{\pi} \int_{0}^{2 \pi} \Delta g(\psi \theta) \cos \theta d \theta=C_{1}(\psi),
$$

and

$$
\frac{1}{\pi} \int_{0}^{2 \pi} d g(\psi \theta) \sin \theta d \theta=S_{1}(\psi),
$$

$\xi$ and $\eta$ are given by

$$
\xi=\frac{1}{2 g} \int_{0}^{\pi} \frac{C_{1}(\psi)}{\psi} d \psi,
$$

and

$$
\eta=\frac{1}{2 g} \int_{0}^{\pi} \frac{S_{1}(\psi)}{\psi} d \psi
$$

If, by the reason already stated, the integration with respect to $\psi$ is extended up to $\psi$ instead of up to $\pi,(28)$ and (29) reduce to

$$
\begin{aligned}
& \xi=\frac{1}{2 g} \int_{0}^{\psi} \frac{C_{1}(\psi)}{\psi} d \psi, \\
& \eta=\frac{1}{2 g} \int_{0}^{\psi} \frac{S_{1}(\psi)}{\psi} d \psi .
\end{aligned}
$$

$a \psi$ is our $r$. Thus (30) and (31) may be written as

$$
\begin{aligned}
& \xi=\frac{1}{2 g} \int_{0}^{r} \frac{C_{1}(r)}{r} d r, \\
& \eta=\frac{1}{2 g} \int_{0}^{r} \frac{S_{1}(r)}{r} d r,
\end{aligned}
$$

which are nothing but the expressions already obtained in (12) and (13).

Proceeding to the second approximation, if the term $\frac{3}{2 \psi}$ in the expression (23) is retained, $\xi$ and $\eta$ are given

$$
\begin{aligned}
\xi & =\frac{1}{2 g} \int_{0}^{\psi} C_{1}(\psi)\left(\frac{1}{\psi}+\frac{3}{2}\right) d \psi \\
& =\frac{1}{2 g} \int_{0}^{r} C_{1}(r)\left(\frac{1}{r}+\frac{3}{2 a}\right) d r, \\
\eta & =\frac{1}{2 g} \int_{0}^{\psi} S_{1}(\psi)\left(\frac{1}{\psi}+\frac{3}{2}\right) d \psi \\
& =\frac{1}{2 g} \int_{0}^{r} S_{1}(r)\left(\frac{1}{r}+\frac{3}{2 a}\right) d r .
\end{aligned}
$$

The following is the summary of what have been sta'ed,

a) in the first approximation,

$$
\begin{aligned}
& \xi=\frac{1}{2 g} \int_{0}^{\infty} \frac{C_{1}(r)}{r} d r, \\
& \eta=\frac{1}{2 g} \int_{0}^{\infty} \frac{S_{1}(r)}{r} d r,
\end{aligned}
$$

b) in the second approximation,

$$
\begin{gathered}
\xi=\frac{1}{2 g} \int_{0}^{\infty} C_{1}(r)\left(\frac{1}{r}+\frac{3}{2 a}\right) d r, \\
\eta=\frac{1}{2 g} \int_{0}^{\infty} S_{1}(r)\left(\frac{1}{r}+\frac{3}{2 a}\right) d r,
\end{gathered}
$$

c) in the third approximation.

$$
\begin{aligned}
& \xi=-\frac{1}{2 g} \int_{0}^{\pi} C_{1}(\psi) \frac{d f(\psi)}{d \psi} \sin \psi d \psi, \\
& \eta=-\frac{1}{2 g} \int_{0}^{\pi} S_{1}(\psi) \frac{d f(\psi)}{d \psi} \sin \psi d \psi
\end{aligned}
$$

The three curves in Fig. 2 show the values of
a) $\frac{1}{2 \pi} \int_{0.1}^{\psi} \frac{1}{\psi} d \psi$
b) $\frac{1}{2 g} \int_{0.1}^{\psi}\left(\frac{1}{\psi}+\frac{3}{2}\right) d \psi$,
c) $-\frac{1}{2 g} \int_{0.1}^{\psi} \frac{d f}{d \psi} \sin \psi d \psi$,

On the other hand, if the earth's radius is $a$, as plotted against $\psi$ or $r$. These are $\xi^{\prime}$ s 


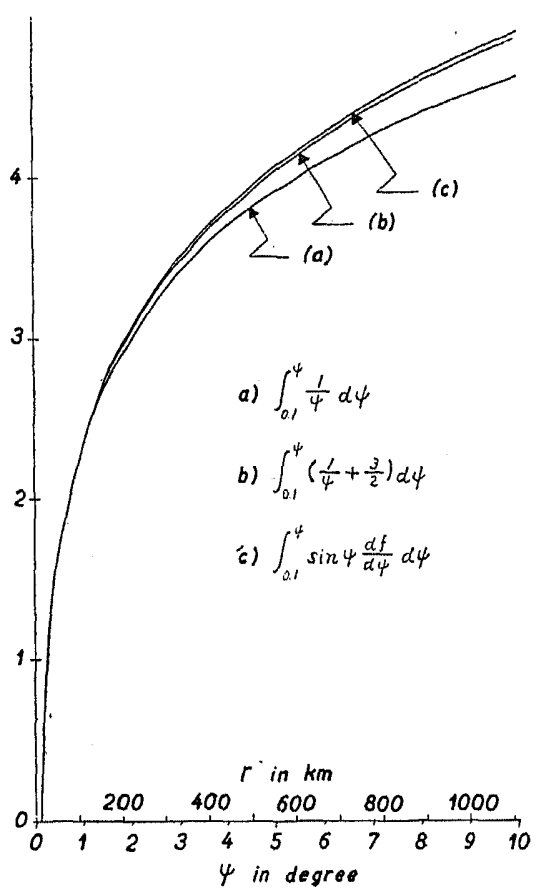

Fig. 2.

when $C_{1}$ or $S_{1}$ are dropped out in (36), (37) and (38). The three curves are very much similar with each other and it will be understood that even when $C_{1}(r)$ and $S_{1}(r)$ do not depend on $r$, the error in the values of $\xi$ and $\eta$ introduced by our first approximation is very small and may safely be ignored.
Thus the conclusion is that the formulas (12) and (13) proposed by the present writers are very satisfactory ones and are widely recommended owing to simplicity of use and reasonable accuracy which can be attained by them. But if one wishes to get more accurate results, the formulas (34) and (35) are to be recommended. These formulas give almost the same values as the exact ones do, provided, of course, $C_{1}(r)$ and $S_{1}(r)$ do not increase much as $r$ increases.

In conclusion, the present writers wish to thank Dr. Donald A. Rice of U S. Coast and Geodetic Survey for kindly placing his unpublished values of $\xi$ and $\eta$ at the 16 American stations at the writers' disposal.

\section{Reference}

Meinesz: F. A. Vening.:

1928 A formula expressing the deflection of the plumb-line in the gravity anomalies and some formula for the gravity-field and gravity-potential outside the geoid.

Kon. Alkad. van Wet. Amesterdam, 31. 315.

RICE: Donald A.:

1952 Deflections of the vertical from gravity anomalies. Bull. géod.. No. 25, 285.

Sollins, A. D. :

1947 Table for the computatien of deflections of the vertical from gravity anomalies. Bull. géod., No. 6, 279. 